\title{
Genetically Divergent Types of the Wheat Leaf Fungus Puccinia triticina in Ethiopia, a Center of Tetraploid Wheat Diversity
}

\author{
J. A. Kolmer and M. A. Acevedo
}

First author: United States Department of Agriculture-Agricultural Research Service Cereal Disease Laboratory, St. Paul, MN 55108; and second author: Department of Plant Pathology, North Dakota State University, Fargo 58108. Accepted for publication 25 December 2015.

\begin{abstract}
Kolmer, J. A., and Acevedo, M. A. 2016. Genetically divergent types of the wheat leaf fungus Puccinia triticina in Ethiopia, a center of tetraploid wheat diversity. Phytopathology 106:380-385.

Collections of Puccinia triticina, the wheat leaf rust fungus, were obtained from tetraploid and hexaploid wheat in the central highlands of Ethiopia, and a smaller number from Kenya, from 2011 to 2013, in order to determine the genetic diversity of this wheat pathogen in a center of host diversity. Single-uredinial isolates were derived and tested for virulence phenotype to 20 lines of Thatcher wheat that differ for single leaf rust resistance genes and for molecular genotypes with 10 simple sequence repeat (SSR) primers. Nine virulence phenotypes were described among the 193 isolates tested for virulence. Phenotype BBBQJ, found only in Ethiopia, was predominantly collected from tetraploid wheat. Phenotype

EEEEE, also found only in Ethiopia, was exclusively collected from tetraploid wheat and was avirulent to the susceptible hexaploid wheat 'Thatcher'. Phenotypes MBDSS and MCDSS, found in both Ethiopia and Kenya, were predominantly collected from common wheat. Phenotypes CCMSS, CCPSS, and CBMSS were found in Ethiopia from common wheat at low frequency. Phenotypes TCBSS and TCBSQ were found on durum wheat and common wheat in Kenya. Four groups of distinct SSR genotypes were described among the 48 isolates genotyped. Isolates with phenotypes BBBQJ and EEEEE were in two distinct SSR groups, and isolates with phenotypes MBDSS and MCDSS were in a third group. Isolates with CCMSS, CCPSS, CBMSS, TCBSS, and TCBSQ phenotypes were in a fourth SSR genotype group. The diverse host environment of Ethiopia has selected and maintained a genetically divergent population of $P$. triticina.
\end{abstract}

Puccinia triticina Erikss., the causal agent of wheat leaf rust, is widespread in wheat-growing regions throughout the world. The disease is most severe in regions where wheat matures in a warm to hot climate such as the continental climates of the Great Plains of North America and the steppes of Central Asia, and also in regions with warm and humid conditions such as coastal regions in North America, South America, and Europe. The most prevalent host of $P$. triticina is common hexaploid (AABBDD genome) bread wheat (Triticum aestivum $\mathrm{L}$.) that is grown around the world. Collections of $P$. triticina from T. aestivum at both local (German and Kolmer 1994) and national (Kolmer et al. 2012) levels are highly variable for virulence to leaf rust resistance genes in wheat and for molecular diversity. Typically, in the United States, 50 to 60 different races or virulence phenotypes of $P$. triticina collected from bread wheat are described on an annual basis. Simple sequence repeat (SSR) DNA markers have been used to genotype $P$. triticina (Duan et al. 2003; Szabo and Kolmer 2007) isolates for molecular variation. In North America (Ordoñez and Kolmer 2009) and other continental populations (Kolmer and Ordoñez 2007; Kolmer et al. 2011; Ordoñez et al. 2010), distinct groups of isolates with identical or highly similar SSR genotypes and virulence phenotypes were described. Because susceptible alternate hosts for P. triticina are not widely distributed throughout most the world, the rust reproduces by the clonal production of urediniospores. On a worldwide level, most SSR virulence phenotype groups of $P$. triticina are distinct due to the effects of mutation, genetic drift, and host selection. However, isolates with identical or similar SSR genotypes and virulence phenotypes have been found in widely separated countries in recent years, indicating the migration of some genotypes between continents (J. A. Kolmer, unpublished data).

Corresponding author: J. Kolmer; E-mail address: Jim.Kolmer@ars.usda.gov

http://dx.doi.org/10.1094/PHYTO-10-15-0247-R

This article is in the public domain and not copyrightable. It may be freely reprinted with customary crediting of the source. The American Phytopathological Society, 2016.
P. triticina is also found on tetraploid (AABB genome) durum wheat (T. turgidum subsp. durum (Desf.) Husn.), which is cultivated in dryer regions compared with common hexaploid wheat. The $P$. triticina isolates virulent to durum wheat have distinct virulence phenotypes that are avirulent to most of the leaf rust resistance genes found in common wheat (Goyeau et al. 2006; Ordoñez and Kolmer 2007b). P. triticina isolates collected from durum wheat are also less variable for virulence and SSR genotypes compared with isolates collected from common wheat (Goyeau et al. 2011; Ordoñez and Kolmer 2007a, b). In recent years, leaf rust has increased on durum wheat in different parts of the world due to the emergence of new phenotypes with increased virulence to resistant durum cultivars (Goyeau et al. 2011; Singh et al. 2004).

Tetraploid wheat is long established in Ethiopia. Emmer wheat (T. dicoccon Shrank), which is the source of the AB genomes in durum and common wheat, was introduced to the central highlands of Ethiopia via the Nile River from Egypt and Sudan over 5,000 years ago (Zaharieva et al. 2010). Vavilov (1951) considered Ethiopia a center of diversity for tetraploid wheat, because he found a number of different varieties there in 1927. In recent years, common wheat has become more prevalent in Ethiopia, accounting for $60 \%$ of wheat production in 2010 (Wheat Atlas 2015); however, landraces of durum wheat and emmer wheat are still grown in the central and northern highlands. The objective of this research was to assess the genetic diversity of $P$. triticina in the central highlands of Ethiopia. We wished to determine whether the uniquely heterogeneous host environment of tetraploid and hexaploid wheat in Ethiopia has selected and maintained a diverse population of $P$. triticina, and whether virulence phenotypes and SSR genotypes found in this population were also present in other worldwide populations.

\section{MATERIALS AND METHODS}

Collections of $P$. triticina were obtained from durum wheat and common wheat in research plots of the Debre Zeit Agricultural 
Research Center in the Oromia region state, $60 \mathrm{~km}$ east of Addis Ababa, in 2011 and 2012. In all, 15 collections were made in 2011 and 14 in 2012. Six collections were also made in research plots at Njoro, Kenya in 2011. In 2013, collections were made in Debre Zeit and at 17 other locations in the central highlands of the Amhara, Oromia, and Southern Nations, Nationalities, and Peoples states (Fig. 1). In total, collections were made from 10 common wheat cultivars and 23 durum wheat and emmer wheat cultivars. The leaves with uredinial infections were placed in paper envelopes and then dried at room temperature. The collections were sent to the United States Department of Agriculture-Agricultural Research Service Cereal Disease Laboratory, St. Paul, MN, where they were stored at $4^{\circ} \mathrm{C}$ until processed for virulence phenotype identification.

Urediniospores from the collections were vacuumed into 00 gel capsules, using small cyclone spore collectors (Browder 1971). The gel capsules were filled with $0.25 \mathrm{ml}$ of Soltrol (Philips Petroleum) nonphytotxic paraffinic oil; then, the oil-spore mixture was atomized onto a single small pot with 20 to 30 7- to 8-day-old seedlings of 'Little Club' wheat, if the collections came from a common hexaploid wheat. If the collections came from a durum wheat or emmer wheat, then 7- to 8-day-old seedlings of the susceptible durum wheat 'RL6089' were used. The Little Club and RL6089 seedlings had been treated with a solution of maleic hydrazide (approximately $30 \mathrm{ml} /$ pot of a solution of $1 \mathrm{~g}$ per 3.0 liters) at coleoptile emergence. Isolation and increase of two to three singleuredinial isolates from each collection from the inoculated Little Club and RL6089 seedlings were as previously described (Kolmer and Hughes 2014) for collections of P. triticina in the annual U.S. virulence survey. Urediniospores of each single-uredinial isolate were inoculated to a 7- to 8-day-old differential set of 20 lines of 'Thatcher' wheat that are near-isogenic for leaf rust resistance genes. Five sets of four differentials were used. Set 1 was lines with genes Lrl (isogenic line RL6003), Lr2a (RL6000), Lr2c (RL6047), and $\operatorname{Lr} 3$ (RL6002); set 2 was lines with $\operatorname{Lr} 9$ (RL6010), Lr16 (RL6005), Lr24 (RL 6064), and Lr26 (6078); set 3 was lines with Lr3ka (RL6007), Lr11 (RL6053), Lr17 (RL6008), and Lr30 (RL6049); set 4 was lines with $\operatorname{LrB}$ (RL6047), Lr10 (RL6004), Lr14a (RL6013), and Lr18 (RL6009); and set 5 was lines with Lr3bg (RL6042), Lr14b

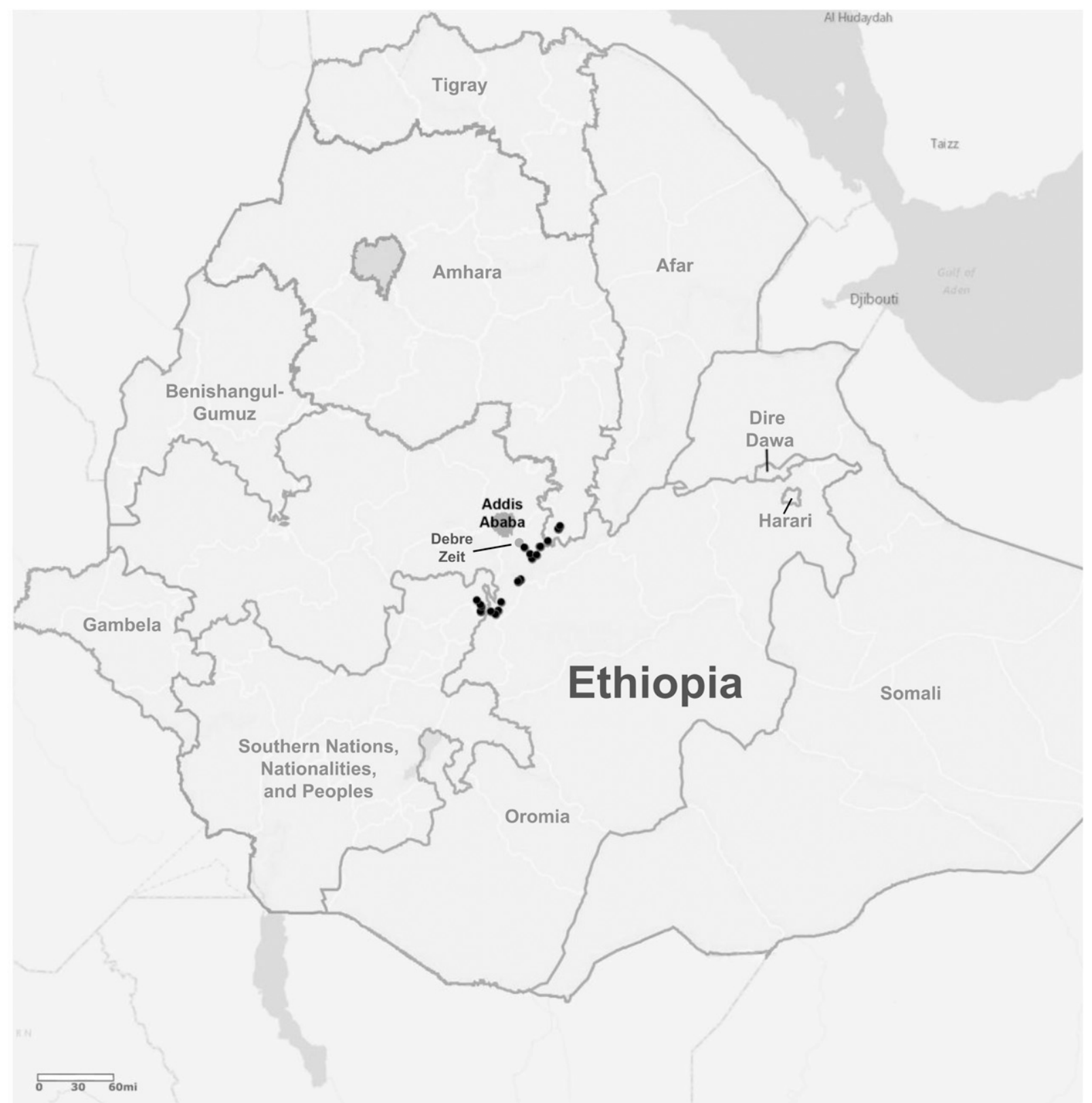

Fig. 1. Map of Ethiopia, indicating sites from which collections of Puccinia triticina were obtained in 2011 to 2013. 
(RL6006), Lr20 (RL 6092), and Lr28 (RL6079). Thatcher was included as a susceptible control. Infection types 0 to $2^{+}$(immune response to moderate uredinia with necrosis or chlorosis) were classified as avirulent and infection types 3 to 4 (moderate to large uredinia without chlorosis or necrosis) were classified as virulent. Each isolate was assigned a five-letter designation based on the hexadecimal code adapted for virulence nomenclature of $P$. triticina in North America (Long and Kolmer 1989). In total, 193 singleuredinial isolates were tested for virulence phenotype.

DNA was extracted from the single-uredinial isolates using the OmniPrep (G-BioSciences) extraction kit. Twenty-three microsatellite pairs (Duan et al. 2003; Szabo and Kolmer 2007) developed from genomic libraries were used to determine the molecular genotypes of 15 isolates that differed for virulence phenotype and had been collected from durum and common wheat. Eight molecular genotypes were described among the 15 isolates, with three distinct groups of molecular genotypes based on two-dimensional principle coordinate plot analysis (PCoA). The 10 primer pairs (68-1, 76-2, 92, 138, 154, $158,164,184,186$, and Fr1) that had the highest number of alleles per locus were chosen to genotype 48 isolates that were selected to represent all virulence phenotypes. Polymerase chain reaction (PCR) amplification and electrophoresis were carried out as previously described (Szabo and Kolmer 2007). Allele sizes in base pairs were scored visually for each primer pair by using a LI-COR 4200 or 4300 DNA sequencer that was calibrated with IRDye 700 molecular-weight size standards. DNA bands generated by each primer pair were standardized with other $P$. triticina isolates previously characterized using the same set of SSR primers.

Isolate genotypes were determined by using molecular weights of alleles at the 10 SSR loci. GENODIVE v2.021b (Meirmans and van Tienderen 2004) was used to determine the total number of SSR genotypes. The SSR genotypes were examined for similarity using a two-dimensional PCoA plot in GENALEX 6.502 (Peakall and Smouse 2006, 2012). Genetic distance was calculated in GENALEX for codominant data between each pair of isolates in an approach based on allelic dosage, as described by Smouse and Peakall (1999). Genetic distance was also calculated in GENODIVE using the clonal distance, which is based on the number of mutation steps between pairs of isolates in a clonal population, and also with Kosman and Leonard's (2005) measure for diploid organisms with codominant markers that is based on allelic identity.

The number of SSR groups was determined by clustering of genotypes in the PCoA plots with each measure of genetic distance. SSR genotype groups were characterized by the average number of alleles per locus, average effective number of alleles per locus, and number of private alleles per locus, as determined with GENALEX. Nei's corrected genetic diversity (Nei 1972) was calculated using GENODIVE and compared with diversity expected under random mating with 999 randomizations of the original dataset.

\section{RESULTS}

Nine different virulence phenotypes of $P$. triticina were found in central Ethiopia and Kenya in the 3 years of sampling (Table 1).
Phenotypes BBBQJ, CCMSS, CCPSS, CBMSS, MBDSS, MCDSS, and EEEEE were collected from Ethiopia and phenotypes MBDSS, MCDSS, TCBSS, and TCBSQ were collected from Kenya. The distributions of the $P$. triticina virulence phenotypes across the different wheat hosts are given in Table 2. Phenotype BBBQJ was virulent only to the Thatcher lines with genes $\operatorname{LrB}, \operatorname{Lr} 10, \mathrm{Lr} 14 \mathrm{~b}$, and Lr20. Phenotypes CCMSS, CCPSS, CMSS, MBDSS, MCDSS, TCBSS, and TCBSS were virulent to several leaf rust resistance genes that are commonly found in hexaploid bread wheat cultivars. Phenotype EEEEE was avirulent to Thatcher wheat; thus, virulence to leaf rust resistance genes in the Thatcher isoline series could not be determined for these isolates. Phenotypes MBDSS and MCDSS were collected predominantly from common wheat, with a smaller number from durum wheat and emmer wheat in Ethiopia and Kenya. In contrast, phenotype BBBQJ was found only in Ethiopia and was collected mostly from durum wheat, with fewer numbers from common wheat and emmer wheat. Phenotypes CCMSS, CCPSS, and CBMSS were found in Ethiopia and collected in relatively equal numbers from common, durum, and emmer wheat. Phenotypes TCBSS and TCBSQ were found in Kenya and were collected from common wheat and durum wheat in close to equal numbers. Isolates with the EEEEE phenotype that were avirulent to seedlings of Thatcher were found only in Ethiopia and were collected exclusively from durum wheat.

In total, 48 isolates were genotyped at the 10 SSR loci (Table 3), with a total of 17 SSR genotypes. Isolates with virulence phenotypes MBDSS and MCDSS had two SSR genotypes and were designated as SSR group ETH-BW1; isolates with phenotypes CCMSS, CCPSS, and CBMSS were identical for SSR genotype and were designated as ETH-BW2; isolates with BBBQJ phenotype had five highly related SSR genotypes and were designated as ETH-BBBQJ; isolates avirulent to Thatcher had eight highly related SSR genotypes and were designated as ETH-EEEEE; and isolates with phenotype TCBSS and TCBSQ were identical for SSR genotype and were designated as KEN-BW (Tables 1 and 3). Isolates in the ETH-EEEEE SSR group were the most diverse overall, with the highest number of SSR genotypes, the highest average number of alleles per locus, and the highest average number of effective alleles per locus. (Table 3). The ETH-EEEEE and ETH-BBBQJ groups had the highest number of private alleles at 11 and 8 , respectively. The observed values of Nei's corrected genetic diversity index for the individual groups and overall were significantly $(P<0.05)$ different than the values expected under random mating.

TABLE 2. Number and virulence phenotypes of Puccinia triticina isolates collected from different wheat hosts in Ethiopia and Kenya from 2011 to 2013

\begin{tabular}{lrrrcrr}
\hline & \multicolumn{5}{c}{ Virulence phenotype } \\
\cline { 2 - 7 } Host & M-DSS & BBBQJ & EEEEE & C-SS & TCBS- & Total \\
\hline Common wheat & 82 & 4 & 0 & 2 & 6 & 103 \\
Durum wheat & 29 & 21 & 33 & 3 & 5 & 81 \\
Emmer wheat & 2 & 2 & 0 & 4 & 0 & 9 \\
Total & 113 & 27 & 33 & 9 & 11 & 193 \\
\hline
\end{tabular}

TABLE 1. Virulence phenotypes and simple-sequence repeat (SSR) genotype groups of Puccinia triticina isolates in Ethiopia and Kenya from 2011 to 2013

\begin{tabular}{|c|c|c|c|}
\hline Virulence phenotype & Virulence & Number of isolates & SSR Group \\
\hline BBBQJ & LrB, Lr10, Lr14b, Lr20 & 27 & ETH-BBBQJ \\
\hline CCMSS & Lr3, Lr26, Lr3ka, Lr30, LrB, Lr10, Lr14a, Lr3bg, Lr14b, Lr20 & 7 & ETH-BW2 \\
\hline CCPSS & Lr3, Lr26, Lr3ka, Lr17, Lr30, LrB, Lr10, Lr14a, Lr3bg, Lr14b, Lr20 & 1 & ETH-BW2 \\
\hline CBMSS & Lr3, Lr3ka, Lr30, LrB, Lr10, Lr14a, Lr3bg, Lr14b, Lr20 & 1 & ETH-BW2 \\
\hline EEEEE & - & 33 & ETH-EEEEE \\
\hline MBDSS & Lr1, Lr3, Lr17, LrB, Lr10, Lr14a, Lr3bg, Lr14b, Lr20 & 33 & ETH-BW1 \\
\hline MCDSS & Lr1, Lr3, Lr26, Lr17, LrB, Lr10, Lr14a, Lr3bg, Lr14b, Lr20 & 80 & ETH-BW1 \\
\hline TCBSS & Lr1, Lr2a, Lr2c, Lr3, Lr26, LrB, Lr10, Lr14a, Lr3bg, Lr14b, Lr20 & 10 & KEN-BW \\
\hline TCBSQ & Lr1, Lr2a, Lr2c, Lr3, Lr26, LrB, Lr10, Lr14a, Lr3bg, Lr14b & 1 & KEN-BW \\
\hline Total & & 193 & \\
\hline
\end{tabular}


The PCoA plots generated by the Smouse and Peakall (1999) genetic distance in GENEALEX, the clonal distance in GENODIVE (Meirmans and van Tienderen 2004), and genetic distance from Kosman and Leonard (2005) were identical for grouping of isolates: the ETH-BW1, ETH-BBBQJ, and ETH-EEEEE isolates were discrete separate groups, while isolates in ETH-BW2 and KEN-BW groups were clustered close together. The PCoA plot based on the Smouse and Peakall distance had the highest percentage of variation $(74 \%)$ explained by the first two dimensions and is given in Figure 2. The PCoA plots based on the clonal distance in GENODIVE and the Kosman and Leonard distance had $70 \%$ of the variation explained by the first two dimensions.

\section{DISCUSSION}

The $P$. triticina population in Ethiopia comprises four highly divergent types that show adaption and virulence to tetraploid durum wheat or hexaploid common wheat. These $P$. triticina types have been selected and maintained by a uniquely heterogeneous and diverse host population. The ETH-EEEEE isolates are virulent to durum wheat, avirulent to Thatcher and other susceptible hexaploid wheat cultivars, distinct for SSR genotype, and have not been found in any other worldwide collections of P. triticina (J. A. Kolmer, unpublished data). $P$. triticina isolates from Ethiopia that were virulent to durum wheat but avirulent to Thatcher wheat were previously noted (Huerta-Espino and Roelfs 1992; Ordoñez and Kolmer 2007b). Coalescence analysis (Liu et al. 2014) of DNA sequence indicated that the ETH-EEEEE isolates were the earliest derived form of $P$. triticina that is pathogenic to either tetraploid or hexaploid wheat. The unique host population structure in Ethiopia may account for the presence of the ETH-EEEEE isolates. Landraces of tetraploid wheat have been grown in Ethiopia for millennia (Payne et al. 2001). Five tetraploid wheat varieties found in 32 wheat landrace populations were described (Eticha et al. 2006): T. durum Desf. (syn. T. turgidum L. subsp. durum), T. turgidum L. (syn. T. turgidum subsp. turgidum) T. aethiopicum Jakubz (syn. T. turgidum L. subsp. durum), T. polonicum L. (syn. T. turgidum L. subsp. polonicum), and T. dicoccon Shrank (syn. T. turgidum L. subsp. dicoccum Thell.). Most of the landrace populations had mixtures of two to three varieties, with a maximum of four varieties in a population. T. durum Desf. was the predominant variety. There was also significant variation within different varieties, because collections of T. durum Desf., T. dicoccon Shrank, and T. turgidum L. were highly variable for microsatellite alleles (Teklu et al. 2006). The low genetic similarity between the three varieties with a number of specific alleles for each variety showed the high genetic diversity in the Ethiopian tetraploid wheat collections. In 1996, emmer (T. dicoccon) was $7 \%$ of Ethiopian wheat production (Zaharieva et al. 2010). The ETH-EEEEE isolates may be a remnant of an early $P$. triticina population that was adapted to tetraploid wheat varieties before the widespread cultivation of common hexaploid wheat. Ethiopian tetraploid landraces are generally highly susceptible to leaf rust (Andenow et al. 1997). If the ETH-EEEEE isolates are long established in Ethiopia on the tetraploid landrace cultivars, then it would be expected that these isolates would have higher levels of genetic diversity compared with other, more recently arrived types.

Isolates of $P$. triticina with high virulence to durum wheat have recently been found and characterized in Mexico (Singh et al. 2004), France (Goyeau et al. 2011), Spain (Martinez et al. 2005), Italy (Mantovani et al. 2010), Israel and Turkey (Kolmer et al. 2011), and Argentina (Ordoñez and Kolmer 2007b). The BBBQJ isolates collected in Ethiopia were identical for virulence and highly similar or identical for SSR genotype compared with the isolates collected from durum wheat in these countries (J. A. Kolmer, unpublished data). These isolates were collected predominantly from durum wheat but were also collected from common wheat cultivars and are virulent to susceptible hexaploid wheat cultivars such as Thatcher, Little Club, and 'Morocco'. In the differential set used for this study, the BBBQJ isolates had virulence only to lines with genes $\operatorname{LrB}, \operatorname{Lr} 10, \mathrm{Lr} 14 b$, and $L r 20$. These genes are all located on the A and B genomes, the same as emmer and durum wheat. The BBBQJ isolates are avirulent to many hexaploid bread wheat cultivars because they have virulence to so few $L r$ genes. Survival and spread of these isolates would be dependent on the presence of durum wheat. Coalescence analysis (Liu et al. 2014) indicated that these isolates were monophyletic and recently derived from $P$. triticina genotypes with virulence to hexaploid wheat. The limited SSR variation of the BBBQJ isolates collected in Ethiopia and the high genotypic similarity with isolates collected in Europe, North America, South America, and the Middle East also suggests a common origin. In 2001, 'Altar C84' durum wheat became susceptible to durum leaf rust in Mexico (Singh et al. 2004). It was later determined that Altar C84 carried Lr72 (Herrera-Foessel et al. 2014) and that many other CIMMYT cultivars and durum germplasm from around the world were also susceptible to this new virulence type of P. triticina. Additional virulence variation to durum wheat cultivars and lines may be present in the BBBQJ isolates from Ethiopia and other countries; however, a differential set of durum wheat genotypes will need to be developed to properly asses this, because the Thatcher differentials do not detect much virulence variation in these isolates. The BBBQJ isolates in Ethiopia may vary for resistance to lines with $L r 72$ or to other durum lines.

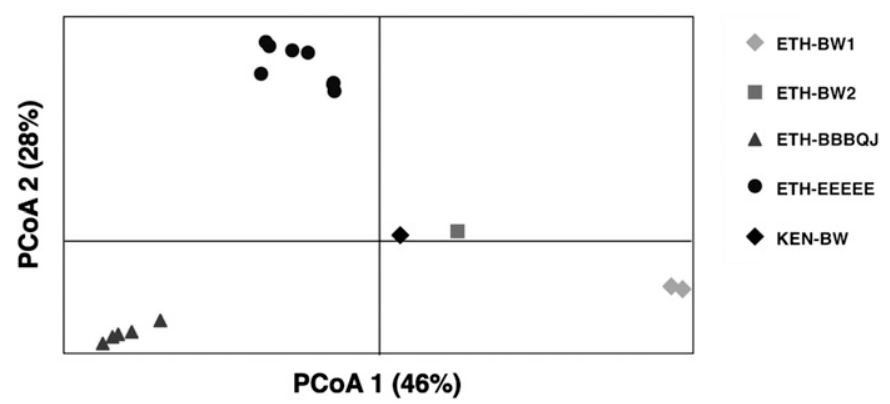

Fig. 2. Principle coordinate plot of 48 isolates of Puccinia triticina from Ethiopia and Kenya grouped using simple-sequence repeat genotypes.

TABLE 3. Genetic diversity of simple-sequence repeat (SSR) genotype groups of Puccinia triticina in Ethiopia and Kenya ${ }^{\mathrm{a}}$

\begin{tabular}{|c|c|c|c|c|c|c|c|}
\hline \multirow[b]{2}{*}{ Group } & \multicolumn{3}{|c|}{ Number of } & \multicolumn{2}{|c|}{ Average number of } & \multirow[b]{2}{*}{ Nei’s ${ }^{b}$} & \multirow[b]{2}{*}{$P^{\mathrm{c}}$} \\
\hline & Isolates genotyped & SSR genotypes & Private alleles & Alleles/locus & Effective alleles/locus & & \\
\hline ETH-BW1 & 15 & 2 & 6 & $1.60(0.22)$ & $1.51(0.17)$ & 0.13 & 0.001 \\
\hline ETH-BW2 & 4 & 1 & 4 & $1.70(0.15)$ & $1.70(0.15)$ & 0.00 & 0.001 \\
\hline ETH-EEEEE & 12 & 8 & 11 & $2.80(0.39)$ & $2.37(0.38)$ & 0.92 & 0.001 \\
\hline KEN-BW & 2 & 1 & 0 & $1.80(0.13)$ & $1.80(0.13)$ & 0.00 & 0.037 \\
\hline Total & 48 & 17 & $\ldots$ & $5.20(0.67)$ & $3.83(0.42)$ & 0.88 & 0.001 \\
\hline
\end{tabular}

${ }^{a}$ Numbers in parentheses indicate standard error.

${ }^{b}$ Nei's corrected genetic diversity.

c Probability of observed Nei's corrected genetic diversity under random mating. 
The isolates with phenotypes MBDSS, MCDSS, CCMSS, CCPSS, and CBMSS are virulent to a number of $L r$ genes and are typical of $P$. triticina isolates with virulence to hexaploid common wheat cultivars. The MBDSS and MCDSS isolates were collected predominantly from common wheat but were also collected from durum wheat. These isolates may have some virulence to durum wheat cultivars, because MBDSS isolates were collected from durum wheat in North Dakota (M. Acevedo, unpublished data) and Chile (J. A. Kolmer, unpublished data). Given that over 100 isolates were characterized from common wheat in this study, it was surprising that only seven virulence phenotypes were described. In other populations of $P$. triticina collected from common wheat, there usually have been a much larger number of virulence phenotypes even from a relatively small geographical area (German and Kolmer 1994; Park et al. 2001). The relative lack of virulence phenotype diversity in Ethiopia may be due to either a recent introduction of a new virulence phenotypes and replacement of previous types, as has recently occurred in European populations of $P$. striiformis (Hovmøller et al. in press), or a strong selection for particular virulence phenotypes by leaf rust resistance genes in the common wheat cultivars. Isolates with MBDSS and MCDSS phenotypes since 1996 have been characterized in North America, South America, South Africa, Europe, the Middle East, and Pakistan. SSR genotypes of the isolates from France, Israel, Turkey, the United States, Canada, Chile, and Uruguay (Kolmer et al. 2011, 2013; Ordoñez and Kolmer 2009; Ordoñez et al. 2010) are identical or highly similar to the SSR genotypes of the MBDSS and MCDSS isolates from Ethiopia (J. A. Kolmer, unpublished data). A worldwide migration of the MBDSS and MCDSS phenotypes has likely occurred in the last 20 years. A set of 66 common wheat cultivars and lines from Ethiopia tested for seedling leaf rust resistance were postulated to have genes $\mathrm{Lrl}, \mathrm{Lr} 10, \mathrm{Lrl} 14 \mathrm{a}, \mathrm{Lr} 23$, $L r 17, L r 24$, and $L r 26$ (J. A. Kolmer, unpublished data). With the exception of $L r 24$, the MBDSS and MCDSS phenotypes are virulent to these $L r$ genes. The predominance of the MBDSS and MCDSS phenotypes may be due to their recent introduction to Ethiopia combined with strong selection for virulence by the common wheat cultivars. Collections of $P$. triticina from 2007 to 2008 in Ethiopia from Debra Zeit were more diverse for virulence, because a number of phenotypes other than MBDSS or MCDSS were detected (Kolmer et al. 2011). Mebrate et al. (2006) determined that $P$. triticina isolates from central Ethiopia, where durum wheat is mostly cultivated, differed for amplified fragment length polymorphism genotype compared with isolates from southern Ethiopia, where common wheat cultivars are more predominant. However, in that study, virulence phenotypes of the isolates were not determined, making it impossible to determine whether the isolates differed for virulence to durum and common wheat.

Isolates with phenotypes CCMSS, CCPSS, and CBMSS were also found in Ethiopia from common and durum wheat, although at much lower frequency. These phenotypes may not be as virulent to the Ethiopian common wheat cultivars as the MBDSS and MCDSS isolates. Isolates with TCBSS and TCBDQ phenotypes were found only in Kenya and were also collected from both common and durum wheat. The C-SS and TCBS-isolates were highly similar for SSR genotype, and had genotypes distinct from the other three groups of $P$. triticina. Sampling of $P$. triticina in regions of Ethiopia outside of the central highlands and more extensive collecting in Kenya may allow the detection of additional virulence phenotypes that were not found in this study. With the increasing cultivation of common wheat and the decreasing importance of durum wheat, it is possible that the virulence diversity of $P$. triticina phenotypes from common wheat will also increase. During the process of wheat domestication and spread of small grain cereal agriculture, the free thrashing durum wheat and common wheat were adopted more rapidly in parts of the Middle East and Europe (Salamini et al. 2002) compared with Ethiopia. In Egypt, which is the likely source of tetraploid wheat in Ethiopia, emmer wheat was the staple crop until the introduction of durum wheat in the Hellenistic period (Oliveira et al. 2012).

In conclusion, a highly divergent population based on SSR genotypes of the wheat leaf rust fungus $P$. triticina is present in a center of diversity for tetraploid wheat in central Ethiopia. The long-term and continued cultivation of tetraploid wheat landraces and the recent increase in cultivation of common hexaploid wheat has allowed a unique mixture of $P$. triticina types to survive. Harlan (1969) attributed the historical diversity of tetraploid wheat and other introduced crops in Ethiopia to the geographic isolation of the fertile central highlands. Other durum-growing regions in the Middle East, Europe, and Mexico are less divergent for $P$. triticina SSR genotypes with virulence to durum wheat because the EEEEE types of isolates are not found in these regions. Liu et al. (2014), using DNA sequence variation in coalescence analysis, hypothesized that the form of $P$. triticina found exclusively on Aegilops speltoides L. in Israel (Ben-Yehuda et al. 2004) was the earliest derived form, followed by the ETH-EEEEE types, followed by the many different genotypes that are adapted to hexaploid wheat which is, by far, the most commonly grown wheat. In this manner, the evolutionary divergence of $P$. triticina parallels the history of genome hybridization in wheat, progressing from a progenitor of the diploid $B$ genome, to tetraploid $\mathrm{AB}$ genomes, and finally to hexaploid $\mathrm{ABD}$ genomes. The $P$. triticina population in central Ethiopia provides a unique opportunity to study diversity and selection in a plant pathogen population that exists in a historical center of host crop diversity.

\section{ACKNOWLEDGMENTS}

We thank K. Xiao, A. Fox, and M. Hughes for excellent technical assistance; B. Girma, G. H. Abraha, and W. Legesse for assistance in obtaining collections of P. triticina; E. Kosman for calculation of genetic distances; and P. Smouse for useful discussions.

\section{LITERATURE CITED}

Andenow, Y., Hulluka, M., Belay, G., and Tesemma, T. 1997. Resistance and tolerance to leaf rust in Ethiopian tetraploid wheat landraces. Plant Breed. 116:533-536.

Ben-Yehuda, P., Eilam, T., Manisterski, J., Shimoni, A., and Anikster, T. 2004. Leaf rust on Aegilops speltoides caused by a new forma specialis of Puccinia triticina. Phytopathology 94:94-101.

Browder, L. E. 1971. Pathogenic Specialization in Cereal Rust Fungi, Especially Puccinia recondita f. sp. tritici: Concepts, Methods of Study, and Application. U. S. Dep. Agric. Agric. Res. Serv. Tech. Bull. No. 1432.

Duan, X., Enjalbert, J., Vautrin, D., Solignac, C., and Giraud, T. 2003. Isolation of 12 microsatellite loci, using an enrichment protocol, in the phytopathogenic fungus Puccinia triticina. Mol. Ecol. Notes 3:65-67.

Eticha, F., Belay, G., and Bekele, E. 2006. Species diversity in wheat landrace populations from two regions of Ethiopia. Genet. Resour. Crop Evol. 53: 387-393.

German, S. E., and Kolmer, J. A. 1994. Virulence Phenotypes of Puccinia recondita f. sp. tritici in Uruguay. Plant Dis. 78:1139-1141.

Goyeau, H., Berder, J., Czerepak, C., Gautier, A., Lanen, C., and Lannou, C. 2011. Low diversity and fast evolution in the population of Puccinia triticina causing durum wheat leaf rust in France from 1999 to 2009, as revealed by an adapted differential set. Plant Pathol. 61:761-772.

Goyeau, H., Park, R., Schaeffer, B., and Lannou, C. 2006. Distribution of pathotypes with regard to host cultivars and French wheat leaf rust populations. Phytopathology 96:264-273.

Harlan, J. R. 1969. Ethiopia: A centre of diversity. Econ. Bot. 23:309-314.

Herrera-Foessel, S. A., Huerta-Espino, J., Calvo-Salazar, V., Lan, C. X., and Singh, R. P. 2014. Lr72 confers resistance to leaf rust in durum wheat cultivar Atil C2000. Plant Dis. 98:631-635.

Hovmøller, M. S., Walter, S., Bayles, R. A., Hubbard, A., Flath, K., Sommerfeld, N., Leconte, M., Czembor, P., Rodriguez-Algaba, J., Thach, T., Hansen, J. G., Lassen, P., Justesen, A. F., Ali, S., and de Vallavielle-Pope, C. Replacement of the European wheat yellow rust population by new races from the centre of diversity in the near-Himalayan region. Plant Pathol. In press. doi:10.1111/ ppa. 12433

Huerta-Espino, J., and Roelfs, A. P. 1992. Leaf rust on durum wheats. Votr. Pflanzenzuchtg. 24:100-102. 
Kolmer, J. A., Hanzalova, A., Goyeau, H., Bayles, R., and Morgounov, A. 2013. Genetic differentiation of the wheat leaf rust fungus Puccinia triticina in Europe. Plant Pathol. 62:21-31.

Kolmer, J. A., and Hughes, M. E. 2014. Physiologic specialization of Puccinia triticina on wheat in the United States in 2012. Plant Dis. 98: 1145-1150.

Kolmer, J. A., Long, D. L., and Hughes, M. E. 2012. Physiologic specialization of Puccinia triticina on wheat in the United States in 2010. Plant Dis. 96:1216-1221.

Kolmer, J. A., and Ordoñez, M. E. 2007. Genetic differentiation of Puccinia triticina populations in Central Asia and the Caucasus. Phytopathology 97: 1141-1149.

Kolmer, J. A., Ordoñez, M. E., Manisterski, J., and Anikster, Y. 2011. Genetic differentiation of Puccinia triticina populations in the Middle East and genetic similarity with populations in Central Asia. Phytopathology 101: 870-877.

Kosman, E., and Leonard, K. J. 2005. Similarity coefficients for molecular markers in studies of genetic relationships between individuals for haploid, diploid and polyploid species. Mol. Ecol. 14:415-424.

Liu, M., Rodrigue, N., and Kolmer, J. 2014. Population divergence in the wheat leaf rust fungus Puccinia triticina is correlated with wheat evolution. Heredity 112:443-453.

Long, D. L., and Kolmer, J. A. 1989. A North American system of nomenclature for Puccinia recondita f. sp. tritici. Phytopathology 79:525-529.

Mantovani, P., Maccaferri, M., Tubersoa, R., and Kolmer, J. 2010. Virulence phenotypes and molecular genotypes in collections of Puccinia triticina from Italy. Plant Dis. 94:420-424.

Martinez, F., Sillero, J. C., and Rubiales, D. 2005. Pathogenic specialization of Puccinia triticina in Andalusia from 1998 to 2000. J. Phytopathol. 153: 344-349.

Mebrate, S. A., Dehne, H. W., Pillen, K., and Oerke, E. C. 2006. Molecular diversity in Puccinia triticina isolates from Ethiopia and Germany. J. Phytopathol. 154:701-710.

Meirmans, P. G., and van Tienderen, P. H. 2004. GENOTYPE and GENODIVE: Two programs for the analysis of genetic diversity of asexual organisms. Mol. Ecol. Notes 4:792-794.

Nei, M. 1972. Genetic distance between populations. Am. Nat. 106:283-292.

Oliveira, H. R., Campana, M. G., Jones, H., Hunt, H. V., Leigh, F., Redhouse, D. I., Lister, D. L., and Jones, M. K. 2012. Tetraploid wheat landraces in the Mediterranean basin: Taxonomy, evolution and genetic diversity. PLoS One 7:e37063.

Ordoñez, M. E., German, S. E., and Kolmer, J. A. 2010. Genetic differentiation within the Puccinia triticina population in South America and comparison with the North American population suggests common ancestry and intercontinental migration. Phytopathology 100:376-383.

Ordoñez, M. E., and Kolmer, J. A. 2007a. Simple sequence repeat diversity of a world-wide collection of Puccinia triticina from durum wheat. Phytopathology 97:574-583.

Ordoñez, M. E., and Kolmer, J. A. 2007b. Virulence phenotypes of a worldwide collection of Puccinia triticina from durum wheat. Phytopathology 97:344-351.

Ordoñez, M. E., and Kolmer, J. A. 2009. Differentiation of molecular genotypes and virulence phenotypes of Puccinia triticina from common wheat in North America. Phytopathology 99:750-758.

Park, R. F., Goyeau, H., Felsenstein, G., Bartos, P., and Zeller, F. J. 2001. Regional phenotypic diversity of Puccinia triticina and wheat host resistance in western Europe, 1995. Euphtyica 122:113-127.

Payne, T. S., Wanjema, J. K., and Girma, B. 2001. Eastern Africa wheat pool. Pages 901-938 in: The World Wheat Book A History of Wheat Breeding. A. Bonjean and W. Angus, eds. Lavoisier, Paris.

Peakall, R., and Smouse, P. E. 2006. GENALEX 6: Genetic analysis in Excel. Population genetic software for teaching and research. Mol. Ecol. Notes 6: 288-295.

Peakall, R., and Smouse, P. E. 2012. GenAlEx 6.5: Genetic analysis in Excel. Population genetic software for teaching and research: An update. Bioinformatics 28:2537-2539.

Salamini, F., Ozkan, H., Brandolini, A., Schafer-Pregl, R., and Martin, W. 2002. Genetics and geography of wild cereal domestication in the near east. Nat. Rev. Genet. 3:429-441.

Singh, R. P., Huerta-Espino, J., Pfeiffer, W., and Figueroa-Lopez, P. 2004. Occurrence and impact of a new leaf rust race on durum wheat in northwestern Mexico from 2001 to 2003. Plant Dis. 88:703-708.

Smouse, P. E., and Peakall, R. 1999. Spatial autocorrelation analysis of individual multiallelic and multilocus genetic structure. Heredity 82:561-573.

Szabo, L. S., and Kolmer, J. A. 2007. Development of simple sequence repeat markers for the plant pathogenic rust fungus Puccinia triticina. Mol. Ecol. Notes 7:708-710.

Teklu, Y., Hammer, K., Huang, X. Q., and Roder, M. S. 2006. Analysis of microsatellite diversity in Ethiopian tetraploid wheat landraces. Genet. Resour. Crop Evol. 53:1115-1126.

Vavilov, N. I. 1951. The origin, variation, immunity, and breeding of cultivated plants. Pages 37-39 in: Selected writings of N. I. Vavilov. K. S. Chester, ed. The Ronald Press Company, New York.

Wheat Atlas. 2015. Online reference. http://wheatatlas.org/

Zaharieva, M., Ayanna, N. G., Hakimi, A. A., Misra, S., and Monneveux, P. 2010. Cultivated emmer wheat (Triticum dicoccon Schrank), an old crop with promising future: A review. Genet. Resour. Crop Evol. 57:937-962. 